\title{
Influence of Postoperative Posture on Macular Slippage after Macula-Off Retinal Detachment: A Randomized Controlled Trial
}

\author{
Megir Schawkat - Christophe Valmaggia - Corina Lang • \\ Hendrik P. N. Scholl · Steven Harsum • Ivo Guber · Josef Guber (D)
}

Received: June 26, 2019 / Published online: August 8, 2019

(C) The Author(s) 2019

\begin{abstract}
Introduction: To evaluate the effect of postoperative posture on the retinal shift after retinal detachment repair.

Methods: Patients who underwent pars plana vitrectomy (PPV) for macula-off rhegmatogenous retinal detachment (RRD) were included prospectively in the current study. Patients were
\end{abstract}

Enhanced digital features To view enhanced digital features for this article go to https://doi.org/10.6084/ m9.figshare.8943500.

M. Schawkat · C. Valmaggia · C. Lang · J. Guber (₫) Eye Clinic, Cantonal Hospital Sankt Gallen, St. Gallen, Switzerland e-mail: josef.guber@kssg.ch

H. P. N. Scholl

Institute of Molecular and Clinical Ophthalmology Basel (IOB), Basel, Switzerland

H. P. N. Scholl · J. Guber

Department of Ophthalmology, University of Basel, Basel, Switzerland

H. P. N. Scholl

Wilmer Eye Institute, Johns Hopkins University, Baltimore, USA

S. Harsum

Epsom and St Helier University Hospitals, NHS

Trust, London, UK

I. Guber

Department of Ophthalmology, University of

Geneva, Geneva, Switzerland randomized into two groups: group A included patients who did a log roll postoperatively, and group B included patients who had to lie flat on their backs for $6 \mathrm{~h}$ postoperatively before moving into the end position. Patients in group A and patients in group B were reviewed after 3 weeks and after 6 weeks, respectively, and fundus autofluorescence images (FAF) were obtained to visualize the retinal rotation.

Results: The sample included 50 eyes from 49 patients. Retinal shift occurred after RRD repair in 17 patients (34\%). There was no statistically significant difference between the two groups $(p=0.94)$. Postoperative macular shift occurred significantly less often $(p=0.049)$ in participants in whom heavy fluid was used in the procedure. Metamorphopsia was reported postoperatively by 10 of 17 patients with retinal shift $(p<0.001)$.

Conclusion: In our study, postoperative posture did not significantly influence postoperative macular slippage after RRD repair. The use of intraoperative heavy liquid appears to be associated with a lower occurrence of retinal shift.

Keywords: Macular shift; Pars plana vitrectomy; Posture; Retinal detachment

\section{INTRODUCTION}

Pars plana vitrectomy (PPV) has become a standard procedure for rhegmatogenous retinal 
detachment (RRD) with favorable outcomes [1-3]. Unfortunately, despite the successful reattachment of the retina achieved using this procedure, various studies have shown that up to $70 \%$ of patients experience macular slippage postoperatively [4]. Macular shift can lead to metamorphopsia and severe binocular diplopia [4-10]. Furthermore, younger patients are more likely than older patients to complain of distorted vision [8]. In view of this, factors and methods that reduce macular slippage following RRD repair should be investigated.

Macular slippage can be visualized using fundus autofluorescence (FAF) images as hyperfluorescent lines [5]. It is believed that those hyperfluorescent lines indicate the locations of retinal vessels preoperatively $[4,11]$. Using this diagnostic tool, macular shift has also been observed preoperatively in patients with idiopathic epiretinal membrane or after macular hole surgery and epiretinal membrane peeling [12-14].

Retinal shift following RRD repair has been reported in several studies [4-10]. Here, we report the first prospective study to compare the occurrence of retinal shift following macula-off RRD repair between two postoperative postures. The aim of this study was to compare two common postoperative posture regimes in order to prevent macular shift after RRD repair.

\section{METHODS}

The local ethics committee approved the study, which was undertaken based on departmental quality controls (Ethikkommission Ostschweiz, BASEC number 2018-00104). Research conformed to the principles of the 1964 Helsinki Declaration and its later amendments or comparable ethical standards. Informed consent was obtained from all individual participants included in the study.

Patients who presented with a macula-off retinal detachment at our eye clinic from June 2017 to June 2018 were included prospectively. The study excluded patients suffering from other forms of retinal detachment such as exudative or tractional. Furthermore, patients with proliferative vitreoretinopathy, retinal redetachments, and patients who needed a primary oil fill were excluded. Two experienced vitreoretinal surgeons (CL, JG) performed the surgeries. Patients were randomly assigned to one of two cohorts using numbered containers in sequence, where the patients had to perform a log roll postoperatively (for patients assigned to group A) or to lie flat on their backs for at least $6 \mathrm{~h}$ before moving into the end position (for patients assigned to group B). A log roll was defined as $30 \mathrm{~min}$ face to temporal followed by $30 \mathrm{~min}$ face down. In each group, the final position was taken according to the retinal break, and the final position was maintained for about 7 days postoperatively.

Each patient received a complete ophthalmological examination prior to surgery with slit-lamp biomicroscopy through a dilated pupil, including best-corrected visual acuity (BCVA) using the Snellen visual acuity chart (noted in logMAR). An Amsler grid was used for all patients to detect the presence of postoperative metamorphopsia. Autofluorescence imaging (FAF) and spectral domain optical coherence tomography (SD-OCT) were performed postoperatively at 3 weeks in $20 \%$ sulfur hexafluoride $\left(\mathrm{SF}_{6}\right)$ gas and at 6 weeks in $12 \%$ perfluoropropane $\left(\mathrm{C}_{3} \mathrm{~F}_{8}\right)$ gas. The Spectralis HRA + OCT (from Heidelberg Engineering, Germany, with a barrier filter of $500 \mathrm{~nm}$ and an excitation wavelength of $488 \mathrm{~nm}$ ) was used to record the images. Evidence of retinal displacement was based on increased autofluorescence lines parallel to retinal vessels. A compass was used to manually measure the amount of retinal shift in degrees (MS, verified by JG) (Fig. 1).

The data collected included the gas used $\left(\mathrm{C}_{3} \mathrm{~F}_{8}\right.$ or $\mathrm{SF}_{6}$ gas), the intraoperative application of perfluorocarbon liquid (PFCL), the number of quadrants with RRD, lens status (pseudophakic, phakic), macula involvement, side located (right or left), and demographics. The primary outcome measure was whether the type of posture influenced the occurrence of postoperative macular shift. Secondary outcome measures were the use of heavy liquid, the type of gas used, the number of quadrants detached, and the age of the patient. 


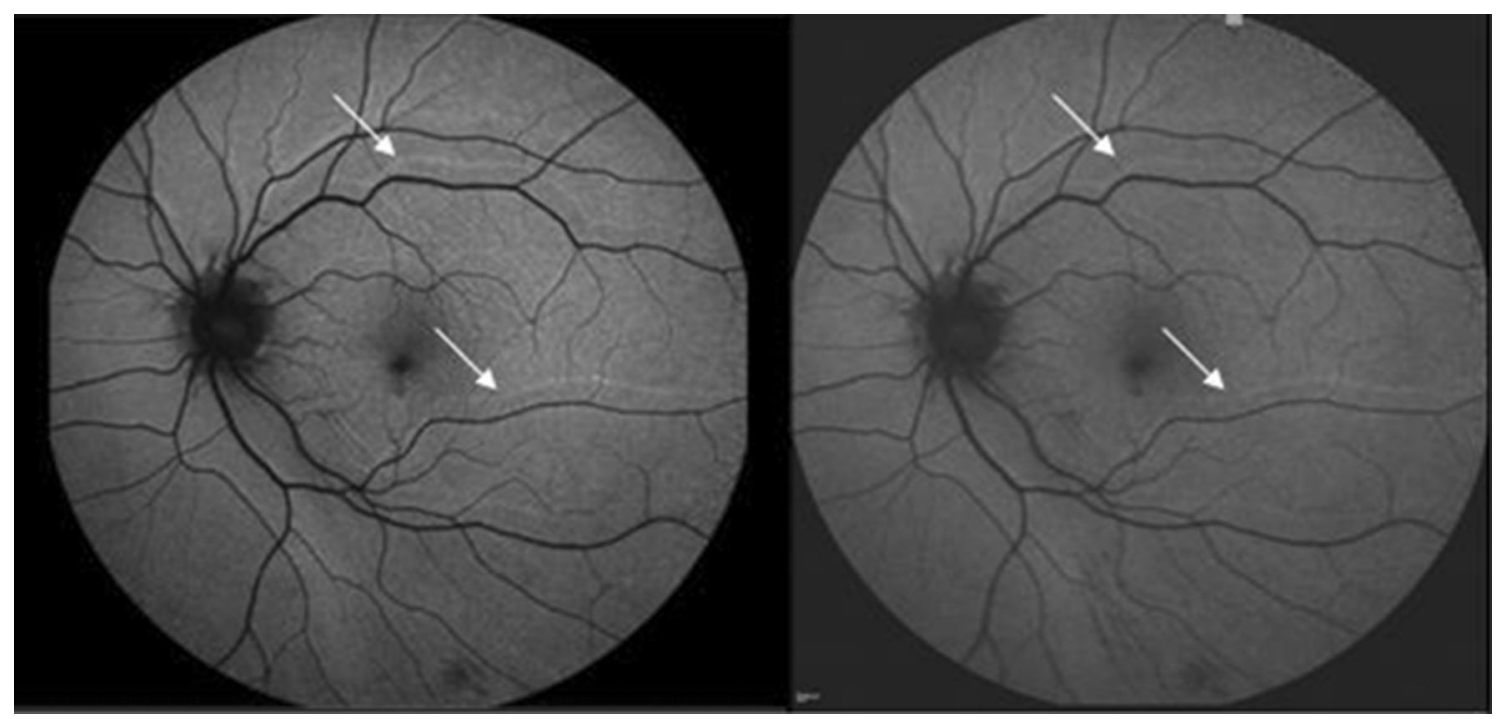

Fig. 1 FAF 3 weeks after surgery (left) and 8 weeks after surgery (right). The preoperative position of the retinal vessel is illustrated by the white arrows pointing to

\section{Surgical Technique}

A standard core and peripheral 3-port PPV (23gauge; Stellaris, Bausch + Lomb, New York, USA) was performed in all patients. Surgery was done with general anesthesia. After the vitrectomy and separation of the posterior hyaloids using the suction method, retinal reattachment was achieved directly through fluid-air exchange with subretinal fluid drainage via main peripheral breaks or through perfluorooctane liquid (Okta-line, Bausch + Lomb) followed by fluid-air exchange. Adjuvant posterior retinotomies were not performed. Retinopexy was done using endolaser or transconjunctival cryocoagulation. 12\% Perfluoropropane $\left(\mathrm{C}_{3} \mathrm{~F}_{8}\right)$ or $20 \%$ sulfur hexafluoride $\left(\mathrm{SF}_{6}\right)$ gas tamponade was applied after the surgery. Rhegmatogenous retinal detachment characteristics determined the selection of intraocular tamponade. Nevertheless, in principle, an injection of $\mathrm{SF}_{6}$ gas was employed for retinal breaks within the upper 240 retinal degrees, whereas an injection of $\mathrm{C}_{3} \mathrm{~F}_{8}$ gas was used when inferior retinal tears appeared and for patients with a low-compliance posture. For phakic eyes, PPV and phacoemulsification were conducted in a single procedure. hyperfluorescent lines. The hyperfluorescent lines were still visible and did not change position in the 6 weeks following surgery

Postoperatively, patients were asked to perform initial posturing according to randomization before moving into the end position.

\section{Statistical Analysis}

A sample size calculation was performed before starting the trial. This calculation considered the patients needed for a $90 \%$ power to detect a significant difference in nonzero rotation between two cohorts by assuming frequencies of $10 \%$ versus $50 \%$. The resulting required sample size was $n=23$ per cohort.

All analyses were carried out with $\mathrm{R}$ version 3.2.3 (2015-12-10). Because the outcome "postoperative rotation" (measured in degrees) had a strongly skewed distribution, rotation was primarily analyzed as the binary outcome of rotation (any rotation $>0^{\circ}$ ) or no rotation. The percentage and number of patients with rotation were recorded for all groups to be compared. Fisher's exact test was used to assess the significance of differences in retinal rotation between groups. To calculate the joint effects of PFCL use and type of posture on the presence of a postoperative shift, a multiple logistic regression model was used. Logistic regression was applied to test relations with numeric predictors 
(number of quadrants with RRD, age). Furthermore, the mean retinal rotation in degrees \pm SD was registered for patients that had rotations $>0^{\circ}$, and $t$ tests were applied to check for a significant difference in degree of rotation between groups. Finally, both aspects (degree and presence of rotation) were jointly tested by obtaining the median and range of degree of retinal rotation within each posture group and comparing both groups using the Wilcoxon test. Pre- to postoperative changes in visual acuity and retinal shift were compared between patients with and without metamorphopsia. The median and range of visual acuity (in logMAR) in each patient cohort were calculated and both groups were compared using the Wilcoxon test.

\section{RESULTS}

A total of 50 eyes from 49 patients (36 men, 14 women) with a macula-off RRD were used in the study. The median age of the patients was 69 years (range 43-90). Phacoemulsification was performed together with PPV in 32\% (16 eyes). Table 1 summarizes the characteristics of the patients at baseline.

Twenty-six patients (52\%) were randomized into the log-roll group (A) while 24 patients $(48 \%)$ were randomized into the lie-flat group (B). The retinal shift on fundus autofluorescence imaging (FAF) showed retinal displacement in 17 patients (34\%). The retinal shifts ranged between $1.0^{\circ}$ and $7.5^{\circ}$.

Using a multiple logistic regression model, the type of posture was found to have no impact on the incidence of macular shift $(p=0.94)$. Furthermore, when rotation occurred, the degree of retinal shift was similar in both groups $(p=1.0)$. When heavy liquid was used in the procedure, a significantly smaller postoperative macular shift was recorded $(p=0.049)$. The number of quadrants detached and the type of gas used had no influence on postoperative outcomes. Younger patients ( $<60$ years) were found to be at greater risk of retinal slippage postoperatively $(p=0.03)$ (Table 2$)$.

There was found to be a significant relationship between postoperative metamorphopsia
Table 1 Patient characteristics

\begin{tabular}{|c|c|c|}
\hline Characteristics & $n=50$ & $\%$ \\
\hline \multicolumn{3}{|l|}{ Sex } \\
\hline Female & 14 & 28 \\
\hline Male & 36 & 72 \\
\hline \multicolumn{3}{|l|}{ Side } \\
\hline Left & 25 & 50 \\
\hline Right & 25 & 50 \\
\hline \multicolumn{3}{|l|}{ Quadrants detached } \\
\hline One & 2 & 4 \\
\hline Two & 23 & 46 \\
\hline Three & 17 & 34 \\
\hline Four & 8 & 16 \\
\hline Phacoemulsification & 16 & 32 \\
\hline \multicolumn{3}{|l|}{ PFCL } \\
\hline Yes & 25 & 50 \\
\hline No & 25 & 50 \\
\hline \multicolumn{3}{|l|}{ Tamponade } \\
\hline $\mathrm{SF}_{6}$ gas & 44 & 88 \\
\hline $\mathrm{C}_{3} \mathrm{~F}_{8}$ gas & 6 & 12 \\
\hline \multicolumn{3}{|l|}{ Posture } \\
\hline Log roll & 26 & 52 \\
\hline Flat & 24 & 48 \\
\hline
\end{tabular}

and retinal shift. Ten of 17 patients with postoperative retinal shift complained of distorted vision $(p<0.001)$; nevertheless, metamorphopsia did not significantly influence overall visual acuity (pre- to postoperative change in logMAR visual acuity: without metamorphopsia, 0.2; with metamorphopsia, $0.3, p=0.75$ ).

\section{DISCUSSION}

Numerous studies have shown that retinal shift can easily be visualized with FAF [4-10, 13]. The 
Table 2 Associations between 5 predictors and the occurrence of postoperative rotation

\begin{tabular}{|c|c|c|c|c|c|c|}
\hline \multirow{2}{*}{ Predictors } & \multirow[t]{2}{*}{ Groups } & \multicolumn{2}{|c|}{ Number (\%) of patients with rotation } & \multirow{2}{*}{$\begin{array}{l}\text { Fisher's test } \\
p \text { value }\end{array}$} & \multirow{2}{*}{$\begin{array}{l}\text { Mean rotation } \\
\left(\text { if } \text { rot }>0^{\circ}\right)\end{array}$} & \multirow{2}{*}{$\begin{array}{l}t \text { test } \\
\left(\text { if rot }>0^{\circ}\right)\end{array}$} \\
\hline & & No $\left(0^{\circ}\right)$ & Yes $\left(>0^{\circ}\right)$ & & & \\
\hline \multirow[t]{2}{*}{ Posture } & Log roll & 14 & $12(46.2 \%)$ & $0.94^{*}$ & $3.3 \pm 2.1$ & $t=0.0$ \\
\hline & Lie flat & 19 & $5(20.8 \%)$ & & $3.3 \pm 1.2$ & $p=1.00$ \\
\hline \multirow[t]{2}{*}{ PFCL } & Yes & 21 & $4(16.0 \%)$ & $0.049^{*}$ & $2.3 \pm 0.4$ & $t=-2.1$ \\
\hline & No & 12 & $13(52.0 \%)$ & & $3.6 \pm 2.0$ & $p=0.06$ \\
\hline \multirow[t]{2}{*}{ Gas } & $\mathrm{SF}_{6}$ & 28 & $16(36.4 \%)$ & 0.65 & $3.4 \pm 1.9$ & n.a. \\
\hline & $\mathrm{C}_{3} \mathrm{~F}_{8}$ & 5 & $1(16.7 \%)$ & & $1.7 \pm 1.9$ & \\
\hline \multirow[t]{3}{*}{ Age } & $<60$ & 7 & $7(50.0 \%)$ & $0.03^{* *}$ & $4.2 \pm 2.1$ & $t=-1.7$ \\
\hline & $60-70$ & 8 & $4(33.3 \%)$ & & $2.7 \pm 1.4$ & $p=0.10^{* *}$ \\
\hline & $>70$ & 18 & $6(25.0 \%)$ & & $2.5 \pm 1.5$ & \\
\hline \multirow[t]{4}{*}{ Quadrants detached } & 1 & 2 & $0(0 \%)$ & $0.86^{* *}$ & n.a. & $t=-0.53$ \\
\hline & 2 & 14 & $9(39.1 \%)$ & & $3.5 \pm 2.2$ & $p=0.60^{* * *}$ \\
\hline & 3 & 12 & $5(29.4 \%)$ & & $3.1 \pm 1.7$ & \\
\hline & 4 & 5 & $3(37.5 \%)$ & & $2.9 \pm 1.6$ & \\
\hline
\end{tabular}

Number and \% of patients with/without rotation in each group are shown, along with the mean \pm SD of the degree of rotation among patients with rotation in each group

The significance of group differences (categorical predictors) and of associations with numeric predictors is shown in the table

* The joint impact of posture and PFCL on the occurrence of postoperative shift according to the multiple logistic regression model

${ }^{* *} p$ value from the logistic regression of rotation (yes/no) against age in years or against number of quadrants

*** Linear regression of rotation angle against age in years or against number of quadrants

hyperfluorescent lines parallel to retinal vessels indicate the initial locations of retinal vessels prior to surgery [15]. Following RRD repair, lines of FAF parallel to retinal vessels were observed when the retina was moved downward under the influence of gravity because of the negligible quantity of leftover subretinal fluid and the application of gas as an intraocular tamponade at the end of surgery [16]. One study described an upward retinal shift wherein silicon oil was used as an intraocular tamponade [9].

To avoid any potential bias, another FAF picture was taken 2 months postoperatively of a patient who showed a retinal shift during the first postoperative visit 3 weeks after surgery. There was no significant difference in retinal shift or the appearance of hyperfluorescent lines
2 months postop (Fig. 1). Nevertheless, as illustrated by the studies of Shiragami et al. and Eri Nitta et al., hyperfluorescent lines may move towards the original retinal vessels over a period of 1 year $[5,13]$.

In the present study, we compared two common postoperative posture regimes. We are aware that every retinal surgeon has their own regime that may differ slightly from those of other retinal surgeons. However, regardless of the posture used, the goal is the same: to prevent retinal shift. The idea of a log roll is to shift the retained fluid out of the macula to the periphery. When asking the patient to lay flat on their back, the surgeon relies on the draining quality of the retinal pigment epithelium and hopes that the retina will naturally find its way 
back to its original position. The incidence of retinal shift in patients who did a log roll following surgery was higher than that in the group who had to lie flat on their backs postoperatively (46.2\% vs. $20.8 \%)$; however, this difference was not found to be statistically significant when a multivariate regression model was used. Compared to other studies, we recorded a moderate to low rate of retinal shift (34\%) [4-10]. No correlation between the number of quadrants with RRD and postoperative retinal shift was observed. This finding contrasts with the results of Codenotti et al. [9]. It is worth mentioning that all of the patients in this study had macula-off RRD.

Surprisingly, the risk for retinal shift was significantly lower if heavy liquid was used during the procedure. This is likely due to the smaller amount of subretinal fluid left behind, which subsequently decreases the risk for macular slippage. On the other hand, younger patients were at greater risk of experiencing retinal shift [17]. This finding probably explains why younger patients complain more often about distorted vision postoperatively [8].

This study is weakened by the short followup period, the lack of a quantitative method to assess metamorphopsia, and the relatively small number of patients. The extent to which retinal shift contributed to metamorphopsia is difficult to determine since a substantial number of patients $(7 / 17 ; 41 \%)$ with retinal shift did not complain of metamorphopsia. However, all patients with retinal shift had a postoperative visual acuity of at least logMAR 0.7 , which did not affect their ability to perceive metamorphopsia.

\section{CONCLUSION}

In conclusion, the current study suggests that postoperative posture does not significantly impact on the prevalence of retinal shift. Reducing the amount of subretinal fluid by using PFCL might reduce the occurrence of macular slippage postoperatively. Furthermore, younger patients are at greater risk of experiencing retinal shift postoperatively.

\section{ACKNOWLEDGEMENTS}

We thank Dr. sc. nat. Sabine Güsewell for her assistance with the statistical analysis, and we thank the participants of the study.

Funding. No funding or sponsorship was received for this study or the publication of this article. The rapid service fee was funded by the authors. All authors had full access to all of the data in this study and take complete responsibility for the integrity of the data and the accuracy of the data analysis.

Authorship. All named authors meet the International Committee of Medical Journal Editors (ICMJE) criteria for authorship for this article, take responsibility for the integrity of the work as a whole, and have given their approval for this version to be published.

Disclosures. Josef Guber, Megir Schawkat, Christophe Valmaggia, Corina Lang, Hendrik Scholl, Steven Harsum, and Ivo Guber have nothing to declare.

Compliance with Ethics Guidelines. The local ethics committee approved this study, which was undertaken based on departmental quality controls (Ethikkommission Ostschweiz, BASEC number 2018-00104). This research conformed to the principles of the 1964 Helsinki Declaration and its later amendments or comparable ethical standards. Informed consent was obtained from all individual participants included in the study.

Data Availability. The datasets generated and/or analyzed during the current study are available from the corresponding author on reasonable request. This study was presented at the EURETINA 2018 Congress in Vienna.

Open Access. This article is distributed under the terms of the Creative Commons Attribution-NonCommercial 4.0 International License (http://creativecommons.org/licenses/ by-nc/4.0/), which permits any noncommercial use, distribution, and reproduction in any medium, provided you give appropriate credit 
to the original author(s) and the source, provide a link to the Creative Commons license, and indicate if changes were made.

\section{REFERENCES}

1. Heimann H, Bartz-Schmidt KU, Bornfeld N, Weiss C, Hilgers R-D, Foerster MH. Scleral buckling versus primary vitrectomy in rhegmatogenous retinal detachment: a prospective randomized multicenter clinical study. Ophthalmology. 2007;114(12): 2142-54.

2. de la Rua ER, Pastor JC, Fernandez I, Sanabria MR, Garcia-Arumi J, Martinez-Castillo V, et al. Noncomplicated retinal detachment management: variations in 4 years. Retina 1 project; report 1 . Br J Ophthalmol. 2008;92(4):523-5.

3. Falkner-Radler CI, Myung JS, Moussa S, Chan RVP, Smretschnig E, Kiss S, et al. Trends in primary retinal detachment surgery: results of a bicenter study. Retina. 2011;31(5):928-36.

4. Lee E, Williamson TH, Hysi P, Shunmugam M, Dogramaci M, Wong R, et al. Macular displacement following rhegmatogenous retinal detachment repair. Br J Ophthalmol. 2013;97(10):1297-302.

5. Shiragami C, Shiraga F, Yamaji H, Fukuda K, Takagishi M, Morita M, et al. Unintentional displacement of the retina after standard vitrectomy for rhegmatogenous retinal detachment. Ophthalmology. 2010;117(1):86-92.

6. Dellomo R, Semeraro F, Guerra G, Verolino M, Cinelli M, Montagnani S, et al. Short-time prone posturing is well-tolerated and reduces the rate of unintentional retinal displacement in elderly patients operated on for retinal detachment. BMC Surg. 2013;13:1-5.

7. Dell'omo R, Scupola A, Viggiano D, Grazia Sammarco $M$, De Turris $S$, Romano $M$, et al. Incidence and factors influencing retinal displacement in eyes treated for rhegmatogenous retinal detachment with vitrectomy and gas or silicone oil. Investig Opthalmol Vis Sci. 2017;58:191-9.

8. Zhou C, Lin Q, Chen F. Prevalence and predictors of metamorphopsia after successful rhegmatogenous retinal detachment surgery: a cross-sectional, comparative study. Br J Ophthalmol. 2016;101:725-9.

9. Codenotti M, Fogliato G, Iuliano L, Querques G, Maestranzi G, Prati M, et al. Influence of intraocular tamponade on unintentional retinal displacement after vitrectomy for rhegmatogenous retinal detachment. Retina. 2013;33(2):349-55.

10. Shiragami C, Fukuda K, Yamaji H, Morita M, Shiraga $\mathrm{F}$. A method to decrease the frequency of unintentional slippage after vitrectomy for rhegmatogenous retinal detachment. Retina (Philadelphia, Pa.). 2014;35:758-63.

11. Dell'Omo R, Mura M, Lesnik Oberstein SY, Bijl H, Tan HS. Early simultaneous fundus autofluorescence and optical coherence tomography features after pars plana vitrectomy for primary rhegmatogenous retinal detachment. Retina. 2012;32(4):719-28.

12. Dell'omo R, Cifariello F, Dell'omo E, De Lena A, Di Iorio $\mathrm{R}$, Filippelli $\mathrm{M}$, et al. Influence of retinal vessel printings on metamorphopsia and retinal architectural abnormalities in eyes with idiopathic macular epiretinal membrane. Investig Ophthalmol Vis Sci. 2013;54:7803-11.

13. Nitta E, Shiraga F, Shiragami C, Fukuda K, Yamashita A, Fujiwara A. Displacement of the retina and its recovery after vitrectomy in idiopathic epiretinal membrane. Am J Ophthalmol. 2013;155:1014-20.

14. Rodrigues I, Lee E, Williamson T. Measurement of retinal displacement and metamorphopsia after epiretinal membrane or macular hole surgery. Retina (Philadelphia, Pa.). 2015;36:695-702.

15. Schmitz-Valckenberg S, Holz FG, Bird AC, Spaide RF. Fundus autofluorescence imaging: review and perspectives. Retina. 2008;28(3):385-409.

16. Pandya VB, Ho I-V, Hunyor AP. Does unintentional macular translocation after retinal detachment repair influence visual outcome? Clin Exp Ophthalmol. 2012;40(1):88-92.

17. Guber J, Schawkat M, Lang C, et al. How to prevent retinal shift after rhegmatogenous retinal detachment repair: a prospective randomized study. Ophthalmol Retina. 2019;3(5):417-21. 